\title{
Aneurisma miliar de Leber associado à síndrome de tração vítreomacular: relato de caso
}

\author{
Leber's miliary aneurysms associated with vitreomacular \\ traction syndrome: case report
}

\author{
Luciano Sólia Násser ${ }^{1}$ \\ Herbert Paulo de Almeida ${ }^{2}$ \\ Leandro Cabral Zacarias ${ }^{3}$ \\ Suel Abujamra ${ }^{4}$ \\ André Marcelo Vieira Gomes ${ }^{5}$
}

\begin{tabular}{|l|}
\hline RESUMO \\
\hline Telangiectasias retinianas são anormalidades vasculares primárias e \\
idiopáticas caracterizadas por dilatações irregulares e incompetência dos \\
vasos retinianos com variados graus de exsudação intra e sub-retiniana. \\
O objetivo desse relato é documentar uma rara associação entre aneurisma \\
miliar de Leber e síndrome de tração vítreomacular bem caracterizada à \\
angiofluoresceinografia e tomografia de coerência óptica. O tratamento \\
realizado foi fotocoagulação com laser de argônio nos aneurismas peri- \\
maculares e cirurgia de vitrectomia posterior via pars plana, o que resultou \\
em melhora consistente da acuidade visual. O caso relatado confirma a \\
importância da tomografia de coerência óptica em estudar a interface \\
vítreorretiniana e suas alterações, o que permitiu abordagem completa da \\
doença em questão.
\end{tabular}

Descritores: Telangiectasia/diagnóstico; Aneurisma/patologia; Vasos retinianos/anormalidades; Angiofluoresceinografia; Fotocoagulação/métodos; Corpo vítreo/patologia; Tomografia de coerência óptica; Adulto; Relatos de casos [Tipo de publicação]

\section{INTRODUÇÃO}

Telangiectasias retinianas são anormalidades vasculares primárias e idiopáticas, unilaterais, de etiologia desconhecida, não familiar ${ }^{(1)}$.

São caracterizadas por dilatações irregulares e incompetência dos vasos retinianos com variados graus de exsudação intrarretiniana e subretiniana, apresentando grande predominância em pacientes do sexo masculino $^{(1)}$.

Coats, em 1908, descreveu uma nova entidade clínica caracterizada pela associação de exsudatos retinianos maciços com anomalias vasculares telangiectásicas ${ }^{(2)}$.

Leber, em 1912, descreveu uma entidade clínica caracterizada por aneurismas miliares múltiplos unilaterais em pacientes jovens do sexo masculino, porém, sem a exsudação maciça sub-retiniana. Em seu trabalho, concluiu que estes casos tratavam-se de manifestações iniciais da doença de Coats $^{(3)}$.

Reese, em 1956, reforçou esta teoria ao descrever um caso em que, após longo acompanhamento, os aneurismas miliares de Leber evoluíram para um caso clássico de doença de Coats e propôs, pela primeira vez, o nome de telangiectasias retinianas ${ }^{(4)}$.

Neste caso, relatamos a rara associação entre aneurismas miliares de Leber e síndrome de tração macular em uma paciente do sexo feminino. 


\section{RELATO DO CASO}

MBS, 45 anos, sexo feminino, branca, natural de São Paulo - SP apresentou-se com queixa de baixa da acuidade visual no olho esquerdo (OE) há dois meses, de instalação súbita. Como antecedentes pessoais, referia hipertensão arterial controlada com propranolol. Negava diabetes melitus. De antecedentes familiares e oftalmológicos, nada digno de nota. Negava hábitos e vícios. Ao exame oftalmológico, a paciente apresentava acuidade visual, pela tabela de Snellen, com a melhor correção OD 20/20 e OE conta dedos a um metro. A biomicroscopia de ambos os olhos revelou cristalino com leve opacidade cortical em AO. O restante do exame mostrava-se dentro da normalidade. A tonometria de aplanação resultou $14 \mathrm{mmHg}$ em AO. O exame fundoscópico mostrou no OD: estreitamento arterial e venoso moderado e no $\mathrm{OE}$ presença de dilatações saculares múltiplas no território artério-venoso com moderada exsudação sub-retiniana temporal à mácula. Observada a presença de dois macroaneurismas na arcada arterial temporal superior além de membrana epirretiniana espessa com moderada tração macular. Vítreo aderido à retina (Figura 1).

A paciente foi submetida ao exame de angiofluoresceinografia que revelou a presença de vários aneurismas ao longo das arcadas temporais e vazamento de contraste na região perimacular temporal. Neovasos não foram observados (Figura 2).

$\mathrm{O}$ exame de tomografia de coerência óptica de retina revelou a presença de uma membrana epirretiniana com tração vítreorretiniana na região macular (Figura 3). Além destes exames, foram solicitados hemograma completo e glicemia de jejum, que estavam dentro da normalidade.

Foi indicada fotocoagulação com laser de argônio nos aneurismas peri-maculares, observando posteriormente uma importante regressão da exsudação sub-retiniana (Figura 4), porém, sem melhora de acuidade visual.

Após discutirmos em detalhes com a paciente sobre os possíveis riscos e benefícios, a mesma foi submetida à cirurgia

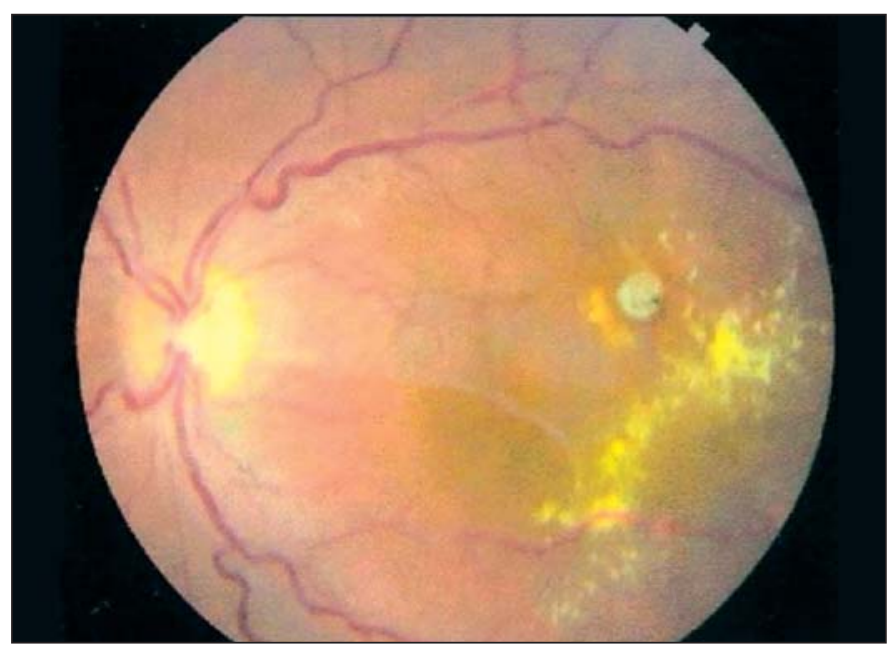

Figura 1 - Fundo de olho esquerdo com múltiplas dilatações saculares, exsudação sub-retiniana e tração vítreomacular de vitrectomia via pars plana associada à remoção de membrana epirretiniana, sem intercorrências intra-operatórias.

A cirurgia resultou em uma considerável melhora do aspecto anatômico da retina (Figuras 5, 6, 7) e uma recuperação

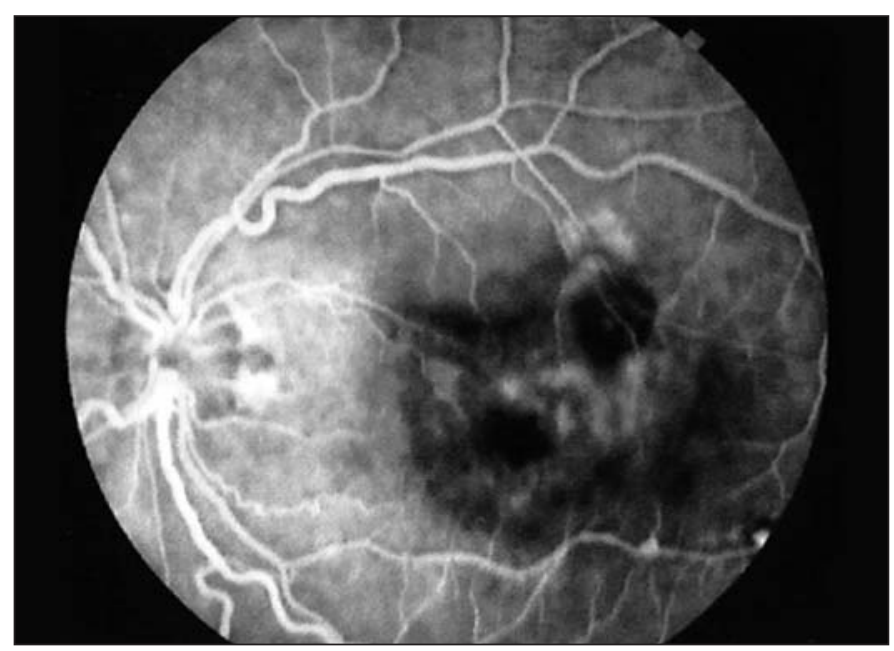

Figura 2 - AFG mostrando vários aneurismas ao longo das arcadas e vazamento de contraste temporal à mácula

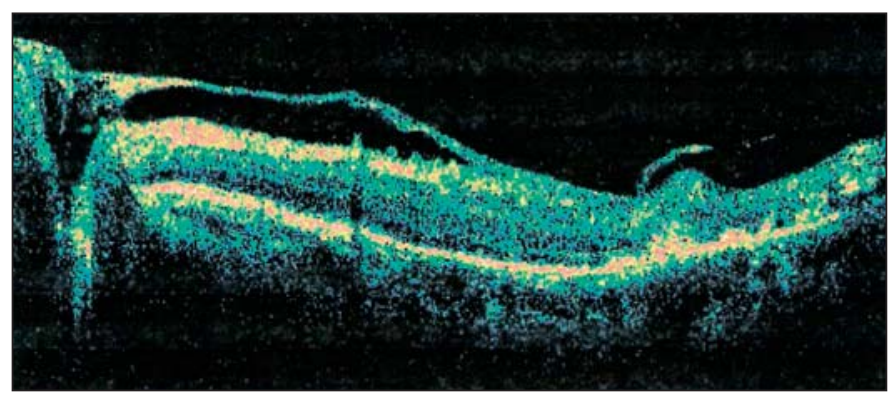

Figura 3 - TCO mostrando presença de membrana epirretiniana com tração vítreorretiniana na região macular

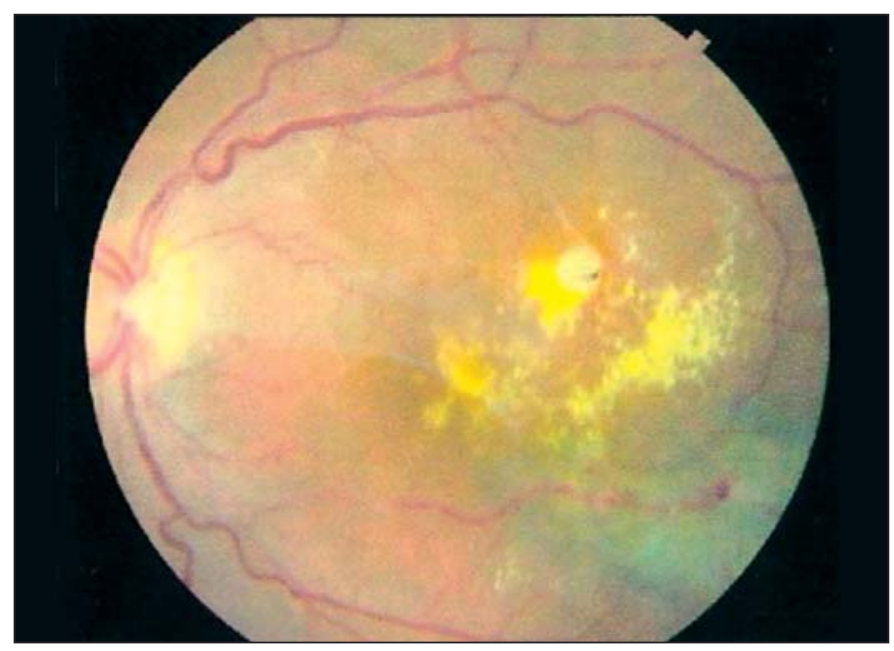

Figura 4 - Fundo de olho esquerdo após laser com regressão da exsudação sub-retiniana 
da acuidade visual no OE para 20/200 c/c, no trigésimo dia de pós-operatório.

\section{DISCUSSÃO}

As telangiectasias retinianas diagnosticadas em adultos apresentam um desafio diagnóstico devido aos vários aspectos que compõem o quadro e às diversas formas de apresentação.

Aneurisma miliar de Leber pode ser o estágio inicial de um mesmo espectro descrito por Coats, quando os fenômenos exsudativos não são tão significativos e as alterações vasculares predominam ${ }^{(5)}$.

Em 1982 Gass classificou as telangiectasias retinianas tardias (média de idade de aparecimento dos sintomas por volta dos 35 anos) com alterações moderadas e limitadas a 2 diâmetros de disco no setor temporal da mácula como telangiectasias justafoveolares unilaterais idiopáticas. Os pacientes acometidos são adultos saudáveis que desenvolvem mo-

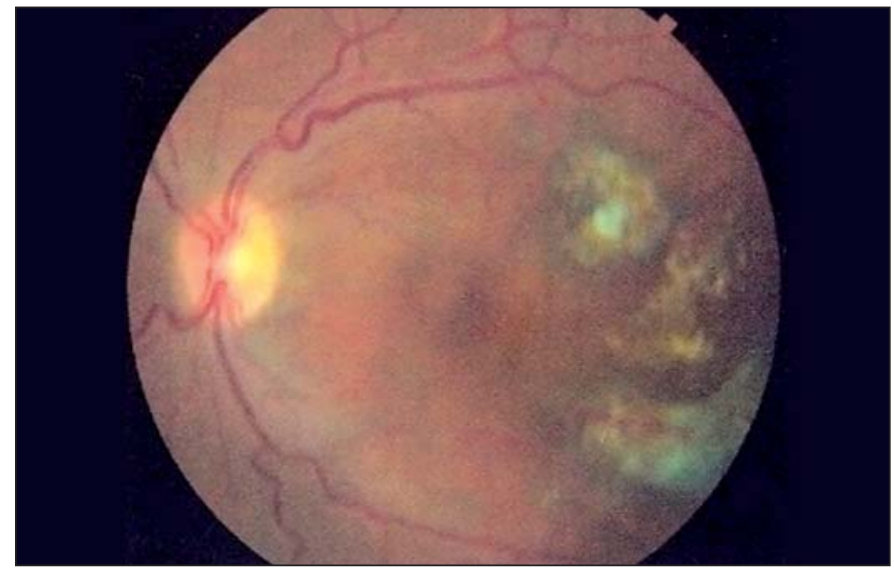

Figura 5 - Fundo de olho após cirurgia mostrando ausência da membrana epirretiniana e regressão da exsudação sub-retiniana

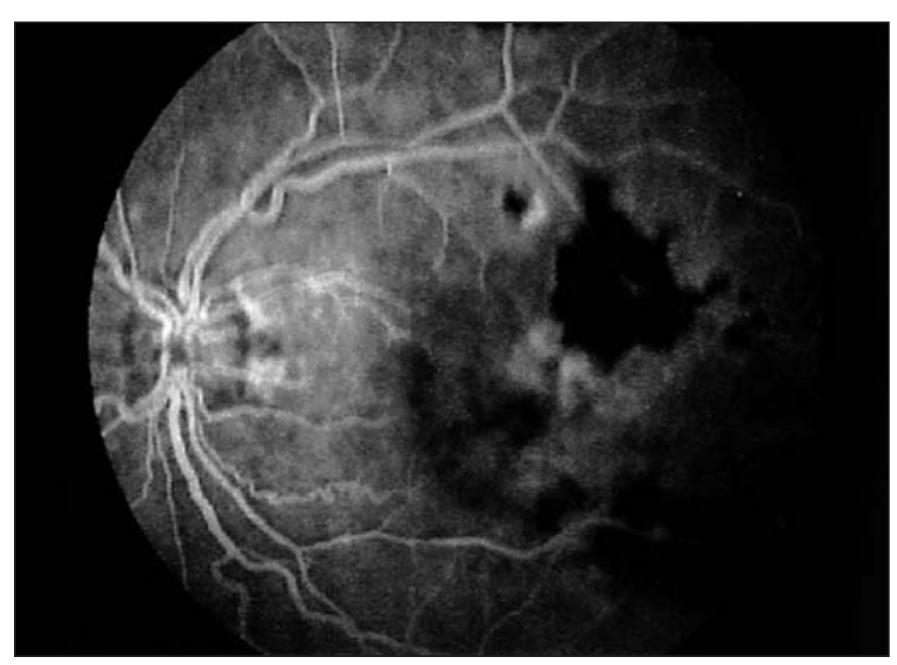

Figura 6 - AFG mostrando ausência de vazamento do contraste e meIhora da arquitetura vascular na mácula

\begin{tabular}{|c|c|c|c|c|}
\hline & Grupo 1A & Grupo 1B & Grupo 2 & Grupo 3 \\
\hline Idade (anos) & 40 & 40 & $50-60$ & 50 \\
\hline Sexo & M & $M$ & Me F & MeF \\
\hline $\begin{array}{l}\text { Área } \\
\text { perifoveolar }\end{array}$ & Temp & $\begin{array}{l}1 \mathrm{~h} \text { de } \\
\text { relógio }\end{array}$ & $\begin{array}{l}\text { Temp ou } \\
\text { total }\end{array}$ & $\begin{array}{c}\text { Obliteração } \\
\text { capilar }\end{array}$ \\
\hline \multicolumn{5}{|c|}{$\begin{array}{l}\text { Temp= região temporal; } 1 \mathrm{~h} \text { do relógio= extensão da área acometida pela } \\
\text { telangectasia, sendo a fóvea o centro do relógio } \\
\text { Fonte: Traduzido de Gass JD, Blodi BA. Idiopathic juxtafoveolar retinal } \\
\text { telangiectasis. Update of classification and follow-up study. Ophthalmology. } \\
\text { 1993;100(10):1536-46. }\end{array}$} \\
\hline
\end{tabular}

derada baixa de visão em um ou ambos os olhos devido à exsudação destes vasos na região macular ${ }^{(5)}$.

Neste mesmo estudo, Gass e Oyakawa baseados em achados angiofluoresceinográficos, classificaram as telangiectasias justafoveolares idiopáticas, classificação atualizada por Gass e Blodi em $1993^{(6)}$ e utilizada até os dias atuais (Tabela 1).

O caso deste relato, por se tratar de uma telangiectasia retiniana numa paciente de 45 anos, unilateral, sem alterações laboratoriais, história familiar e antecedentes pessoais e pelo fato de ser caracterizado mais pelas anomalias microvasculares do que por exsudação intra e sub-retiniana, não se enquadra na classificação de Gass nem na de Coats, tratandose, portanto, de aneurisma miliar de Leber em paciente do sexo feminino, associado a uma síndrome de tração macular, fato não relatado em nenhum dos pacientes estudados por Coats, Leber, Reese e Gass, o que o torna uma rara ocorrência.

A grande maioria dos pacientes com aneurisma miliar de Leber é jovem do sexo masculino ${ }^{(7)}$. Embora as alterações vasculares cursem com um prognóstico favorável, os eventos exsudativos, quando presentes, pioram a qualidade visual ${ }^{(8)}$, fato observado neste paciente.

A síndrome de tração vítreomacular é a condição em que mudanças do corpo vítreo provocam um descolamento posterior e incompleto deste, com persistência de adesões na região macular ${ }^{(9)}$. A maioria das trações vítreomaculares causa distorção na região e quando não ocorre descolamento espontâneo e completo do vítreo, evolui com a formação de uma membrana epirretiniana e subseqüente edema macular cistóide $^{(10)}$. Isto resulta em perda da visão central e meta-

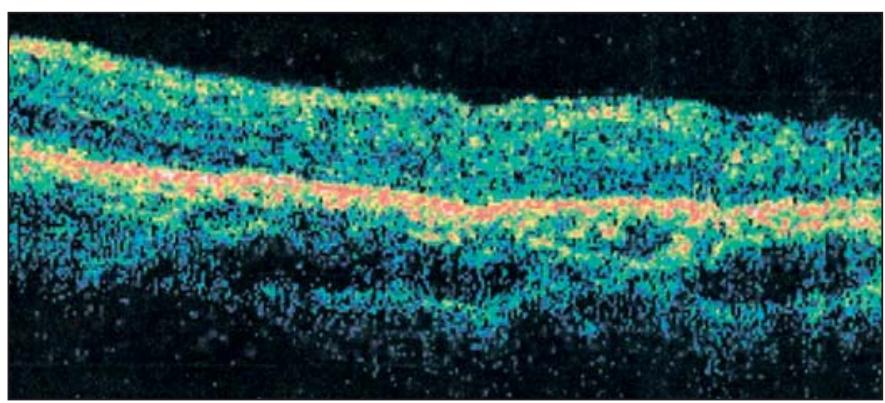

Figura 7 - TCO mostrando recuperação da arquitetura macular, ausência da membrana epirretiniana e do componente tracional 
morfopsia ${ }^{(11)}$, o que explica a baixa visão da paciente, mesmo com a regressão da exsudação pela fotocoagulação (Figura 4).

Por conta deste quadro evolutivo a cirurgia para tratamento da tração vitreorretiniana, visando remover o vítreo e retirar as trações maculares tem mostrado resultado satisfatório nos estudos mais atuais ${ }^{(11)}$, fato reproduzido neste caso.

Embora alguns casos de telangiestasias retinianas regridam espontaneamente, o tratamento com fotocoagulação a laser no intuito de obliterar os vasos telangiectásicos ampliou a margem de sucesso no tratamento ${ }^{(12)}$.

\section{CONCLUSÃO}

O presente caso relata um quadro atípico de aneurisma miliar de Leber em uma mulher de 45 anos, complicado por uma síndrome de tração macular, o que alterou o prognóstico visual da doença.

Apesar do tratamento adequadamente instituído, a acuidade visual não seguiu os resultados dos estudos clássicos e dos mais atuais sobre telangiectasias retinianas, devido à presença de um componente tracional sobre a região macular. A tração vítreorretiniana persistente, mesmo que completamente desfeita cirurgicamente, como foi relatado, deixou seqüelas degenerativas sobre a mácula.

\section{AGRADECIMENTOS}

Ao Dr. Alan Diego Negretto pelas informações acrescentadas ao relato.

\section{ABSTRACT}

Retinal telangiectasias are idiopatic vascular abnormalities of the retina characterizad by irregular dilatation of the retinal vessels, intraretinal and subretinal exsudation. The aim of this article is to describe the uncommon association of Leber's miliary aneurysms and vitreomacular traction syn- drome in a female patient. The diagnosis was established with angiofluoresceinography and optic coherence tomography. The patient was treated with focal photocoagulation with argon green laser directed to the perimacular aneurysms and pars plana posterior vitrectomy. The visual acuity showed great improvement after a four-month follow-up. The present report supports the importance of optic coherence tomography in cases where the vitreoretinal interface must be evaluated, including vascular pathologies, which allowed us to offer a better treatment to this patient.

Keywords: Telangiectasis/diagnosis; Aneurysm/pathology; Retinal vessels/abnormalities; Fluorescein angiography; Light coagulation/methods; Vitreous body/pathology; Tomography, optical coherence; Adult; Case reports [Publication type]

\section{REFERÊNCIAS}

1. Pereira CM, Bonanomi MTBC, Abujamra S. Telangiectasias retinianas. In: Abujamra S, Ávila M, Barsante C, Farah ME, Gonçalves JOR, Lavinsky J, Moreira Júnior CA, Nehemy MB, Susuki H, relatores. Retina e vítreo clínica e cirurgia. São Paulo: Roca; 2000. cap.8. p.551-9.

2. Coats G. Forms of retinal disease with massive exudation. Roy Lond Ophth Hosp Rep. 1907-8;18:440-525.

3. Leber T. Ueber eine durch Vorkommen multipler Miliaraneurysmen charakterisierte Form von Retinaldegeneration. Arch F Ophth. 1912;81:1-14.

4. Reese AB. Telangiectasis of the retina and Coats' disease. Am J Ophthalmol. 1956;42(1):1-8.

5. Gass JD, Oyakawa RT. Idiopathic juxtafoveolar retinal telangiectasis. Arch Ophthalmol. 1982;100(5):769-80.

6. Gass JD, Blodi BA. Idiopathic juxtafoveolar retinal telangiectasis. Update of classification and follow-up study. Ophthalmology. 1993;100(10):1536-46.

7. Schuman JS, Puliafito CA, Fujimoto JG. Optical coherence tomography of ocular diseases. 2nd ed. Thorrofare, New Jersey: Slack; 2004.

8. Smithen LM, Brown GC, Brucker AJ, Yannuzzi LA, Klais CM, Spaide RF. Coats' disease diagnosed in adulthood. Ophthalmology. 2005;112(6):1072-8.

9. Hayasaka S, Katsube T, Yamamoto Y, Setogawa T. Leber's miliary aneurysms in a 63-year-old woman: concurrence of regression and active lesions. Ophthalmologica. 1988;196(4):188-91.

10. Hikichi T, Yoshida A, Trempe CL. Course of vitreomacular traction syndrome. Am J Ophthalmol. 1994;119(1):55-61.

11. Gass JD. Stereoscopic atlas of macular diseases: diagnosis and treatment. $4^{\text {th }}$ ed. St. Louis: Mosby-Year Book; 1997. v.1.

12. Ryan SJ, editor. Retina. $4^{\text {th }}$ ed. St. Louis: Mosby; 2006. v.2. 